\title{
UCRL-PROC-213665
}

LAWRENCE LIVERMORE N A T IO N A L LABORATORY

\section{A Piecewise Linear Finite Element Discretization of the Diffusion Equation for Arbitrary Polyhedral Grids}

T. S. Bailey, M. L. Adams, B. Yang, M. R. Zika

July 18,2005

Mathematics and Computation, Supercomputing, Reactor Physics and Nuclear and Biological Applications Conference (ANS)

Avignon, France

September 12, 2005 through September 15, 2005 
This document was prepared as an account of work sponsored by an agency of the United States Government. Neither the United States Government nor the University of California nor any of their employees, makes any warranty, express or implied, or assumes any legal liability or responsibility for the accuracy, completeness, or usefulness of any information, apparatus, product, or process disclosed, or represents that its use would not infringe privately owned rights. Reference herein to any specific commercial product, process, or service by trade name, trademark, manufacturer, or otherwise, does not necessarily constitute or imply its endorsement, recommendation, or favoring by the United States Government or the University of California. The views and opinions of authors expressed herein do not necessarily state or reflect those of the United States Government or the University of California, and shall not be used for advertising or product endorsement purposes. 


\title{
A Piecewise Linear Finite Element Discretization of the Diffusion Equation for Arbitrary Polyhedral Grids
}

\author{
Teresa S. Bailey and Marvin L. Adams \\ Texas A\&M University \\ Department of Nuclear Engineering \\ College Station, TX 77843-3133 \\ baileyte@tamu.edu,mladams@tamu.edu \\ Brian Yang and Michael R. Zika \\ Lawrence Livermore National Laboratory \\ Livermore, CA 94550 \\ tbyang@llnl.gov
}

\begin{abstract}
We develop a piecewise linear (PWL) Galerkin finite element spatial discretization for the multidimensional radiation diffusion equation. It uses piecewise linear weight and basis functions in the finite element approximation, and it can be applied on arbitrary polygonal (2D) or polyhedral (3D) grids. We show that this new PWL method gives solutions comparable to those from Palmer's finite-volume method. However, since the PWL method produces a symmetric positive definite coefficient matrix, it should be substantially more computationally efficient than Palmer's method, which produces an asymmetric matrix. We conclude that the Galerkin PWL method is an attractive option for solving diffusion equations on unstructured grids.
\end{abstract}

KEYWORDS: diffusion, arbitrary polyhedral grids, piecewise linear finite element

\section{INTRODUCTION}

Several methods have been developed to solve the radiation diffusion equation on arbitrary polyhedral (3D) and polygonal (2D) meshes. A finite-volume (FV) method developed by Palmer $[1,2]$ divides each cell into "corner" subcells and enforces conservation on each dual cell, where a dual cell is the union of corners that touch a vertex. This method is second-order accurate, with relatively small error norms, and exactly reproduces any linear solution. However, Palmer's method does not generate a symmetric coefficient matrix, so the matrix system is (relatively) computationally expensive to store and solve. Wachspress developed rational-polynomial basis functions [3] that can be applied to polyhedral cells in a finite-element method (FEM). His functions have the advantage that a Galerkin FEM formulation will yield a symmetric positive definite (SPD) matrix, but the disadvantage that the basis-function integrals must be done numerically.

In this work we apply a relatively new FEM basis function - the "PieceWise Linear" (PWL) function [4] - to the radiation diffusion equation on arbitrary polygonal and polyhedral meshes. The goals are to gain an SPD coefficient matrix, retain the simplicity and accuracy of Palmer's finite-volume method, and avoid the numerical integrations that plague Wachspress's method. As part of our development we show that Palmer's method is, in fact, a FEM with PWL basis functions, but with non-Galerkin weighting. (The weight functions are constants over dual 
cells.) We compare our Galerkin PWL method against Palmer's method on multiple test problems. We find that our method meets all of out goals: it yields accuracy comparable to Palmer's method, produces an SPD matrix, and involves only very simple integrations.

We remark that this is an active field of research, with methods constantly under development. For example, Morel has proposed a cell-centered FV method for polyhedral cells [5], and support-operator methods have been proposed by Kuznetsov et al. [6]. We do not claim that our PWL FEM is superior to these methods for all problems. Further research is needed to determine which methods are best for different applications.

\section{DEVELOPMENT}

The radiation conservation equation can be written as:

$$
\vec{\nabla} \cdot \vec{F}(r)+\sigma E(r)=S(r)
$$

where $\vec{F}$ is the radiation current, $E$ is the scalar flux, $S$ is the source, and $\sigma$ is the opacity. The current in the diffusion approximation is

$$
\vec{F}=-D \vec{\nabla} E
$$

The diffusion equation is obtained by substituting Eq. (2) into Eq. (1). To apply an FEM to the radiation diffusion equation, we first multiply the equation by a weight function and integrate it over the problem domain. If the $i$-th weight function is nonzero only on cells that touch the $i$-th vertex, then we have

$$
\int_{z a t i} d^{3} r w_{i}(r)[-\vec{\nabla} \cdot D \vec{\nabla} E(r)+\sigma E(r)-S(r)]=0
$$

where $z$ denotes cells (which we sometimes call "zones"). The divergence theorem produces:

$$
\int_{\partial z a t i} d^{2} r w_{i}(r) \vec{n} \cdot \vec{F}+\int_{z a t i} d^{3} r\left[D \vec{\nabla} E(r) \bullet \vec{\nabla} w_{i}(r)+w_{i}(r)(\sigma E(r)-S(r))\right]=0 .
$$

The first term is zero if the cells over which the equation is integrated are not on the boundary of the domain. We ignore it in the remainder of this development. An approximation can be made for $E$ in terms of known basis functions:

$$
E(r)=\sum_{\text {all } j} E_{j} b_{j}(r)
$$

which results in

$$
\sum_{j} E_{j}\left\{\int_{z a t i} d^{3} r\left[D \vec{\nabla} b_{j}(r) \bullet \overrightarrow{\nabla w_{i}}(r)+\sigma b_{j}(r) w_{i}(r)\right]\right\}=\int_{z a t i} d^{3} r w_{i}(r) S(r) .
$$


Eq. (6) is $N$ equations for $N$ unknowns. Given our assumption of one weight function per vertex, $N$ is the number of vertices in the mesh.

A Galerkin FEM sets $w_{i}=b_{i}$. It is not difficult to show that this yields a symmetric positivedefinite matrix. In this work we develop a Galerkin FEM that employs the piecewise linear (PWL) weight and basis functions developed recently by Stone and Adams [4]. In the development of these methods for arbitrary polyhedral cells, we divide each polyhedron into subcells called sides and corners. A side is a tetrahedron made from two adjacent vertices, the zone center, and a face center. A corner is defined as the union of all half-sides that touch a vertex in one zone. Sides are shown in a two dimensional polygonal mesh in figure 1. Figure 2 shows corners on this same mesh.

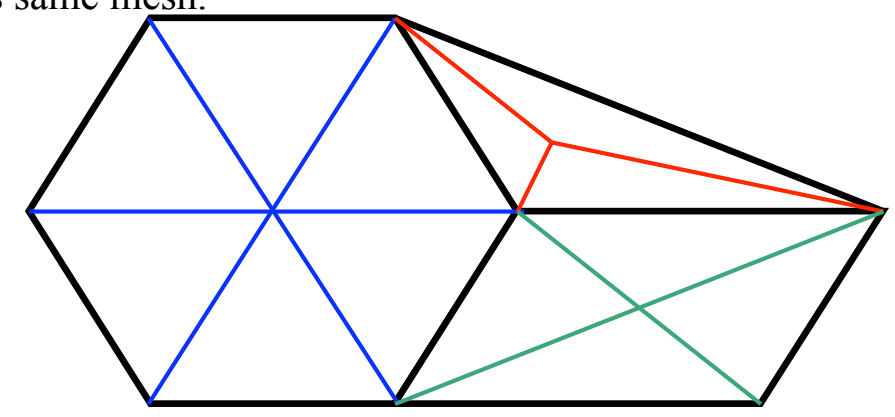

Figure 1: Sides in a 2D polygonal mesh (always triangles in 2D)

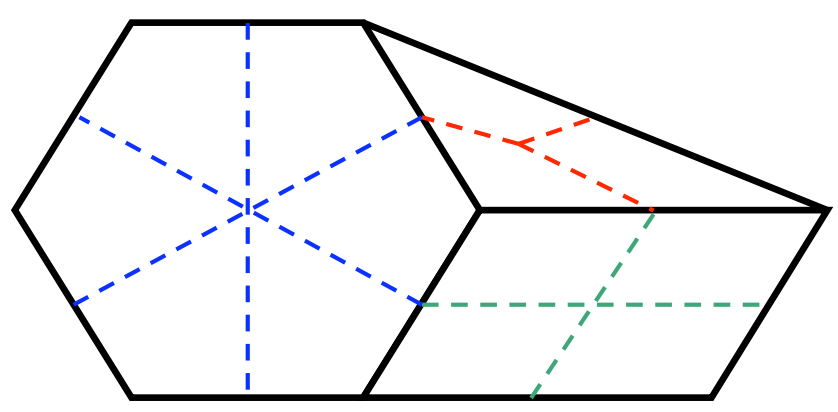

Figure 2: Corners in a 2D polygonal mesh (always quadrilaterals in 2D)

The PWL function centered at vertex $j$ can be written in three dimensions as:

$$
b_{j}(\vec{r})=t_{j}(\vec{r})+\sum_{\text {faces at } p} \beta_{f, j} t_{f}(\vec{r})+\alpha_{z, j} t_{z}(\vec{r}),
$$

where the $t$ functions are standard linear functions defined tetrahedron by tetrahedron. For example, $t_{j}$ equals 1 at the $j$-th vertex and decreases linearly to zero on all other vertices of each side that touches point $j . t_{z}$ is unity at the cell midpoint and zero at each face midpoint and each cell vertex. $t_{f}$ is unity at the face midpoint and zero at the cell midpoint and at each of the face's vertices. The $\alpha_{z}$ and $\beta_{f}$ are weights that give the cell and face midpoints as weighted averages of their vertices: 


$$
\begin{aligned}
& \vec{r}_{z} \equiv \text { cell midpoint }=\sum_{j @ z} \alpha_{z, j} \vec{r}_{j} ; \\
& \vec{r}_{f} \equiv \text { cell midpoint }=\sum_{j @ f} \beta_{f, j} \vec{r}_{j} .
\end{aligned}
$$

Each basis function is piecewise linear on each side, which makes integration over sides straightforward. (Note that the gradient of a basis function is constant on a side.) Figure 3 shows a plot of a PWL basis function for a two-dimensional rectangular cell.

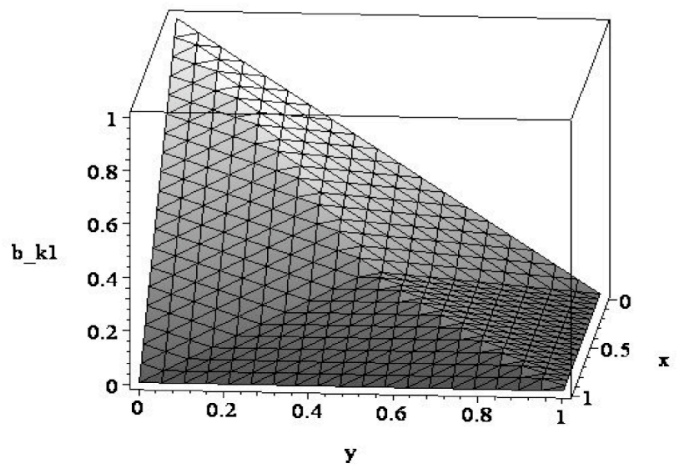

Figure 3: 2D Piecewise Linear Basis Functions Figure courtesy of Hiromi Stone

The definitions in Eqs. (7)-(9) guarantee that any linear function of $(x, y, z)$ can be exactly represented as an expansion in these basis functions. (This subtlety is what makes the method work. Effectively, the functions generate interpolated values at cell and face midpoints, in such a way that the interpolated values are perfect for linear functions.)

Our Galerkin FEM uses PWL functions for the $w$ and $b$ in the general equation above, with one exception: we lump the "mass matrix." That is, we make the replacement:

$$
\int_{z @ i} d^{3} r\left[\sigma b_{j}(r) w_{i}(r)\right] \underset{\text { lump }}{\longrightarrow} \delta_{i j} \int_{c @ i} d^{3} r[\sigma],
$$

where $c$ refers to a "corner" subcell. This defines our method (except for boundary conditions, which are straightforward). In the next section we give numerical results from several test problems.

Palmer's method is developed by dividing each cell into corners, defining dual cells to be the union of corners at each vertex, and enforcing conservation over these dual cells. The gradient of the scalar flux is taken to be constant inside each side subcell, and the cell-center scalar flux is an interpolant of the vertex scalar fluxes, defined such that it is exact for functions that are linear in $x, y$, and $z$. The method enforces continuity of particle flow (the normal component of the current) across each dual cell boundary. See references [1] and [2] for more details.

It is interesting to note that Palmer's FV method is equivalent to an FEM with PWL basis functions and with the mass-matrix lumping defined above, but with weight functions given by: 


$$
w_{i}(\vec{r})= \begin{cases}1, & \vec{r} \in \text { any corner that touches vertex } i, \\ 0, & \text { otherwise. }\end{cases}
$$

As far as we know, this has not been previously observed. This illuminates the close connection between Palmer's method and our Galerkin FEM, and also may permit Palmer's method to be better understood and analyzed.

Both methods should be exact (within the limits of roundoff error) if the solution is a linear function of $x, y$, and $z$, regardless of how distorted the spatial cells become. (We test this below.) This follows from the construction of the PWL basis functions.

\section{RESULTS}

To test our implementation of this method as well as the prediction of perfection for linear solutions, we consider a one-dimensional problem with a linear solution. This problem has no source, no absorption, reflecting boundary conditions for the $z$ and $y$ dimensions, and Dirichlet boundary conditions of $E(0, y, z)=0$ and $E(1, y, z)=1$. Figures 4 and 5 show contour plots of this solution generated by our Galerkin PWL method on a random mesh and a " $Z$-mesh." From these plots (and many, similar ones), we conclude that the PWL method does reproduce the exact linear solution on polyhedral meshes. This property is also attained by Palmer's method. 


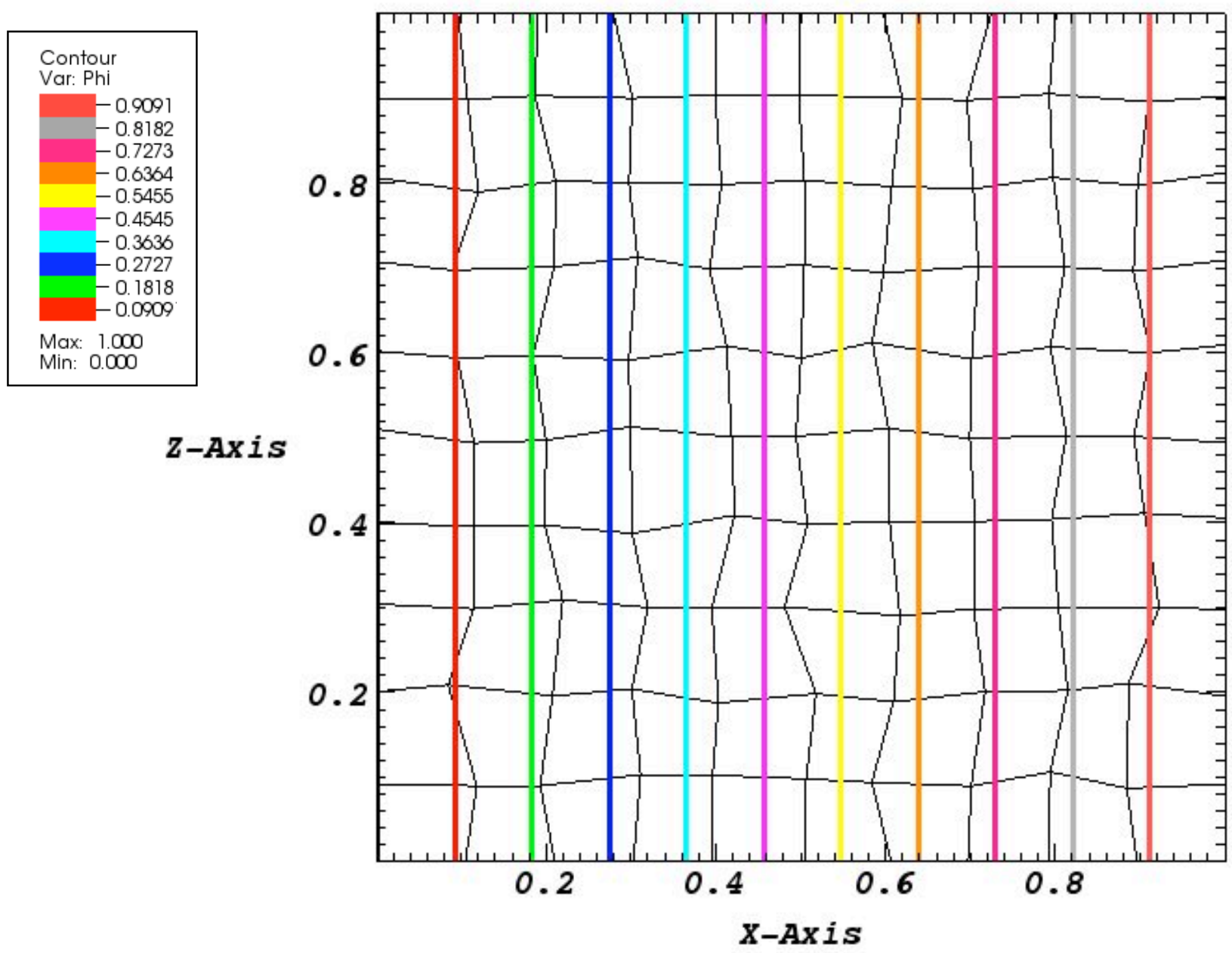

Figure 4: Contour plot of a $1 \mathrm{D}$ linear solution on a random mesh at $y=0.75$. (Solution is the same on other $y$-planes, as it should be.) 

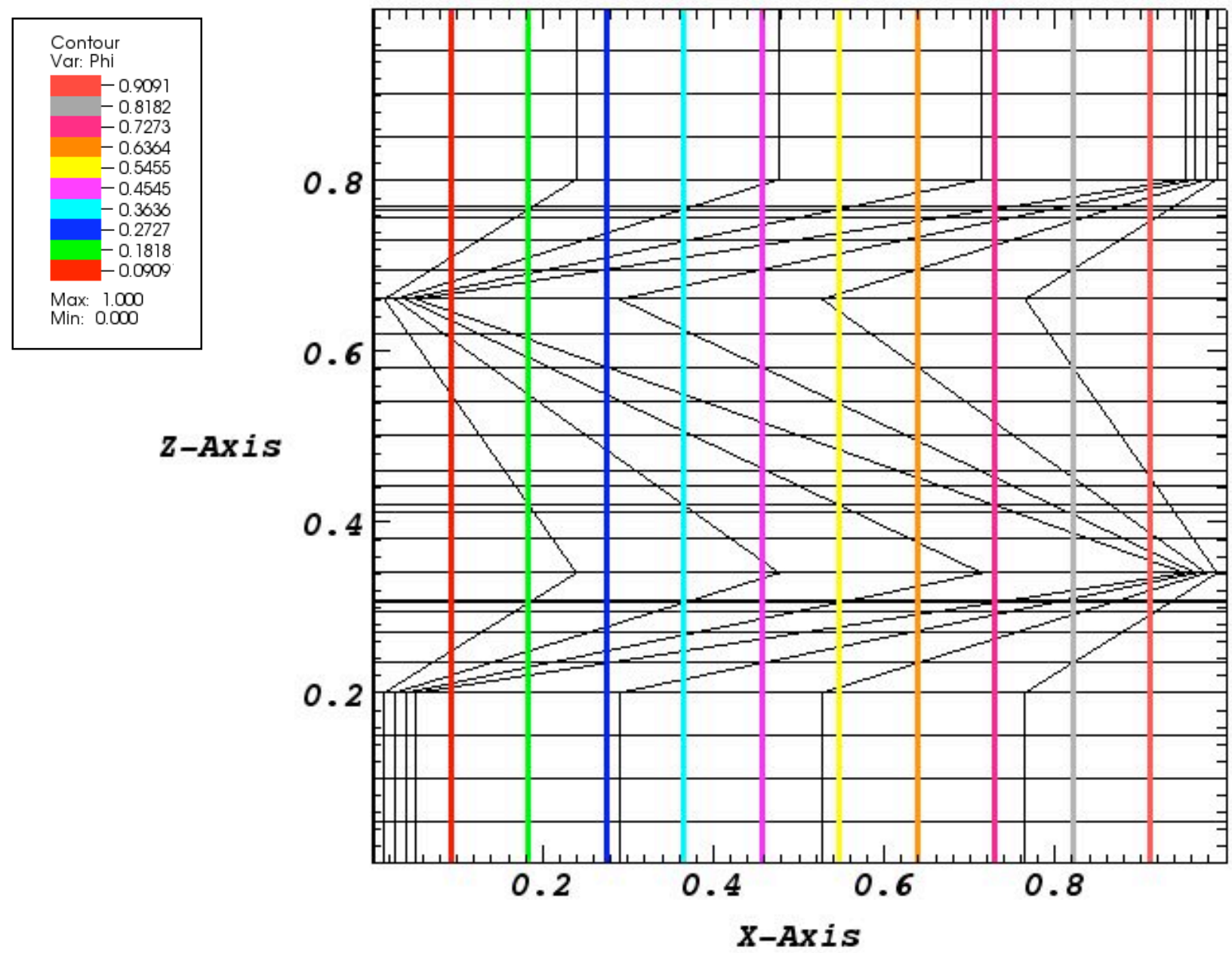

Figure 5: Contour plot of a $1 \mathrm{D}$ linear solution on a $\mathrm{z}$-mesh at $y=0.75$. (Solution is the same on other $y$-planes, as it should be.)

A z-mesh problem with a linear solution in $x, y$, and $z$ was also run to show that the method produces the linear solution on a difficult mesh in all dimensions. This test problem had absorption, a linear source, and applied Dirichlet boundary conditions that enforced a linear solution on each face. Solution contours should be straight diagonal lines, and they are. 

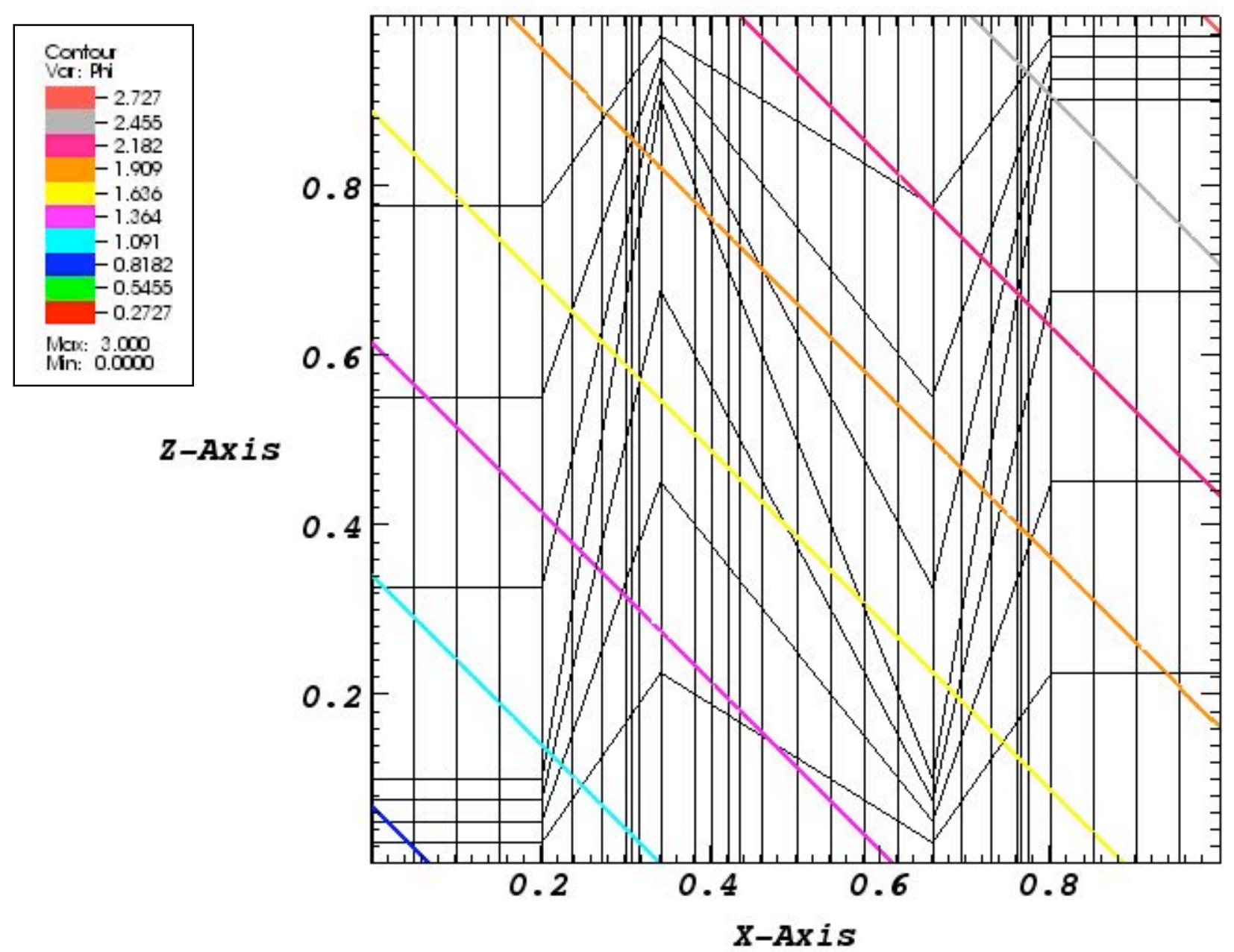

Figure 6: Contour plot of 3D linear solution on a $z$-mesh, slice at $y=0.75$

The next set of problems tests the convergence rate of both the PWL method and Palmer's method on a series of random meshes. (Each mesh was generated by randomly perturbing the vertices in an orthogonal mesh.) One test calculated the convergence rate for a problem with a known one-dimensional quartic solution. This problem has no absorption, a quadratic source, and Dirichlet boundary conditions of $E(0, y, z)=0$ and $E(1, y, z)=1$. The results from this test are found in Table I.

Table I. Convergence rates of Galerkin PWL and Palmer's method for a 1D quartic problem on a series of random meshes

\begin{tabular}{|c|c|c|c|c|}
\hline Random Mesh Size & PWL |Error| & Error Ratio & Palmer |Error| & Error Ratio \\
\hline $2 \times 2 \times 2$ & 0.038431951 & & 0.035921299 & \\
\hline $4 \times 4 \times 4$ & 0.01311606 & 2.930145 & 0.012246572 & 2.933172 \\
\hline $8 \times 8 \times 8$ & 0.003393059 & 3.865557 & 0.003194967 & 3.833083 \\
\hline $16 \times 16 \times 16$ & 0.000928568 & 3.654076 & 0.000877868 & 3.639460 \\
\hline $32 \times 32 \times 32$ & 0.000234858 & 3.953747 & 0.000220536 & 3.980614 \\
\hline $64 \times 64 \times 64$ & $5.892325 \mathrm{E}-05$ & 3.985825 & $5.479132 \mathrm{E}-05$ & 4.025015 \\
\hline
\end{tabular}


The error norm is calculated by taking the L2 norm of the vector of the exact quartic solution minus the calculated solution. The "Error Ratio" in the table is the ratio of the error norm from the previous grid to that from the given grid; perfect second-order convergence will yield an error ratio of 4 . Both methods show second-order accuracy and have the same magnitude of error, although Palmer's method does have slightly smaller error norm values for this problem.

A second test problem was developed to further test the convergence rate. This problem includes absorption, has no source, and Dirichlet boundary conditions of $E(0, y, z)=1$ and $E(1, y, z)=0$. It has an exponential solution. The results from this test are found in Table II.

Table II. Convergence rates of PWL and Palmer's method for a 1D exponential problem on a series of random meshes

\begin{tabular}{|c|c|c|c|c|}
\hline Random Mesh Size & PWL |Error| & Error Ratio & Palmer |Error| & Error Ratio \\
\hline $2 \times 2 \times 2$ & 0.0016012705 & & 0.0016212922 & \\
\hline $4 \times 4 \times 4$ & 0.0005528424 & 2.896432 & 0.0004850215 & 3.342723 \\
\hline $8 \times 8 \times 8$ & 0.0001379511 & 4.007524 & 0.0001300960 & 3.728183 \\
\hline $16 \times 16 \times 16$ & 0.0000362096 & 3.809795 & 0.0000355542 & 3.659093 \\
\hline $32 \times 32 \times 32$ & 0.0000093349 & 3.878966 & 0.0000091722 & 3.876289 \\
\hline $64 \times 64 \times 64$ & 0.0000023968 & 3.894709 & 0.0000023482 & 3.906041 \\
\hline
\end{tabular}

Again, both methods show second-order accuracy behavior and similar errors.

Both convergence-rate test problems were also run on orthogonal "brick" grids. These results are not shown because both methods produce identical, second-order accurate results for one dimensional problems.

We analyzed the Galerkin PWL method in the limit of high aspect ratios on two dimensional orthogonal grids, and found that the signs of the off diagonals will change at certain ratios of $\Delta x$ and $\Delta y$. For $(\Delta x / \Delta y)^{2}<1 / 3$, the sign change occurs for the nodes that are above and below the central node. For $(\Delta y / \Delta x)^{2}>3$, the sign change occurs for the nodes to the left and right of the central node. We ran a test problem on an orthogonal mesh with 64 cells out of 1024 total cells having 1000 to 1 aspect ratios $(\Delta y / \Delta x)$. These cells were contiguous, and inserted into the problem at $\mathrm{x}=0.375$ on a cubical domain of unit width. The test problem had Dirichlet boundary conditions of $E(0, y, z)=0, E(1, y, z)=0, E(\mathrm{x}, 0, z)=0$ and $E(\mathrm{x}, 1, z)=0$, and reflecting boundary conditions in the $\mathrm{z}$ direction. The source in this problem is a "point source" inserted into the problem in the middle of the cells with high aspect ratios. The plot of the PWL solution is shown for an equally spaced mesh in figure 7 and a mesh with high aspect ratio cells in figure 8 . 


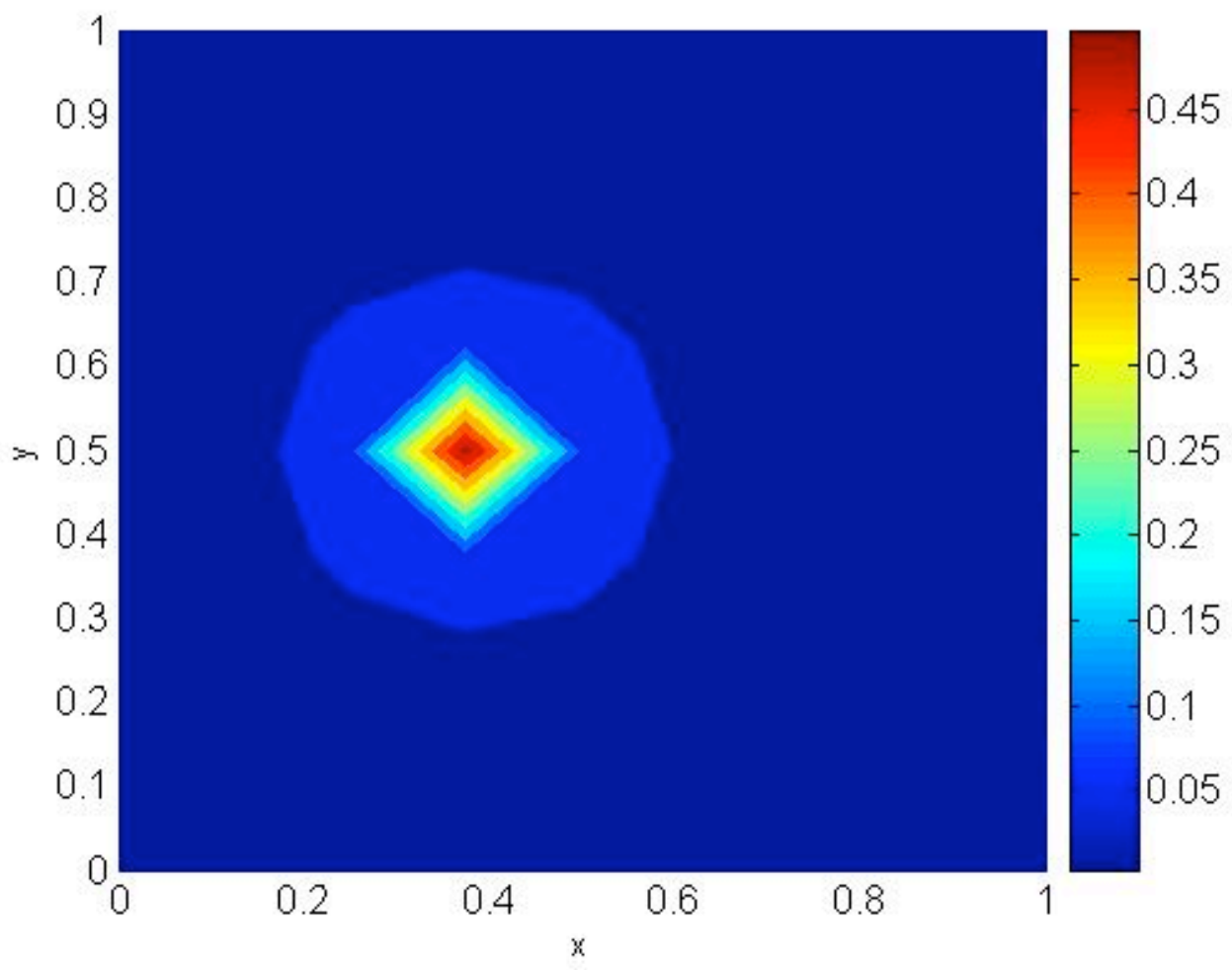

Figure7: Contour plot at $z=0.5$ of the point source problem for an equally spaced mesh with the source at $x=0.375$ and $y=0.5$

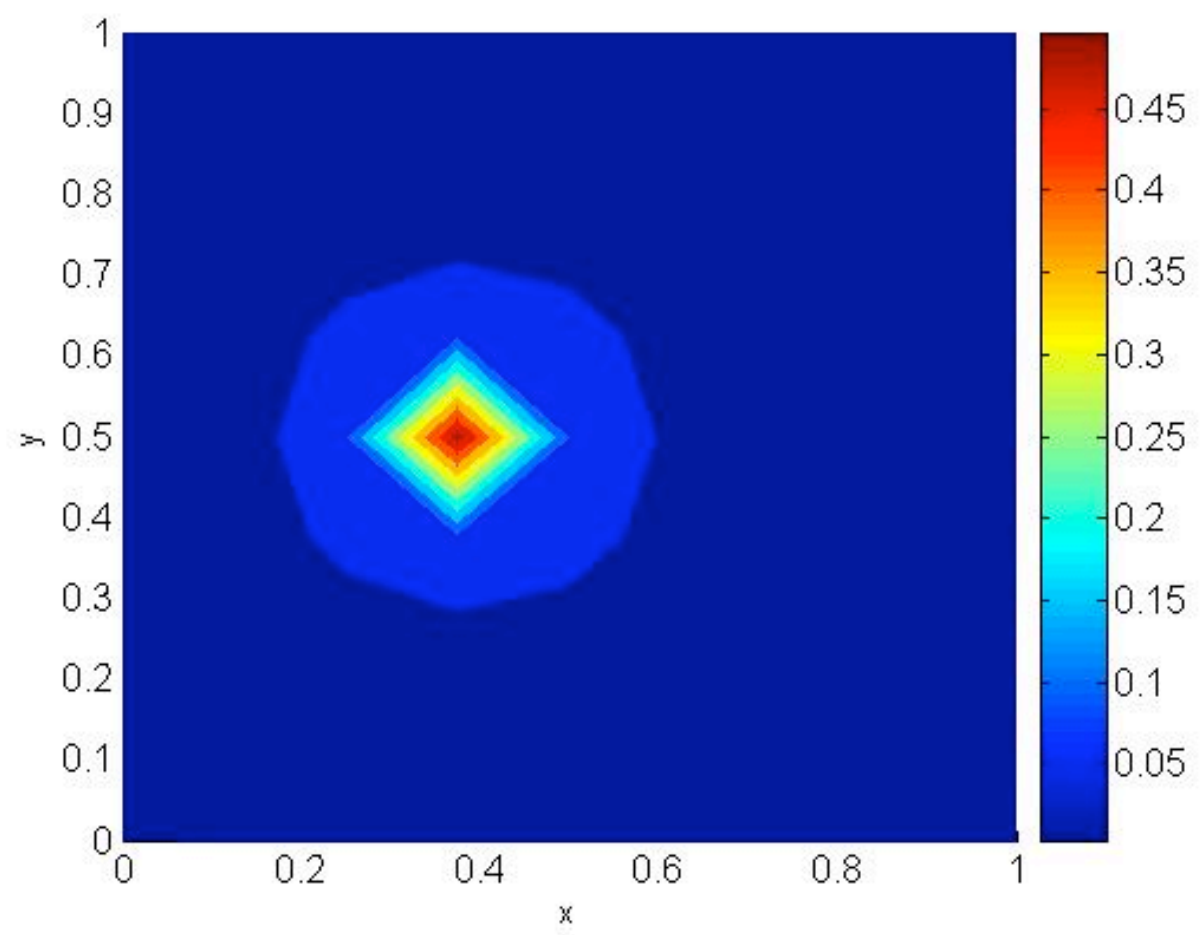

Figure 8: Contour plot at $z=0.5$ of the point source problem for a mesh with high aspect ratio zones with the source at $x=0.375$ and $y=0.5$. 
These plots show just a slice of the problem. The slice was taken in the $\mathrm{z}$ direction at the value of $\mathrm{z}$ where the solution is maximum.

The presence of the high aspect ratio cells does not affect the solution significantly. In particular, despite the singular source, there are no oscillations in the PWL solution. The problems with uniform and non-uniform grids have the same source strength, which is input into both problems at a single node with the same coordinates. The magnitude of the solution is slightly larger $(0.6 \%)$ for the problem with the high aspec-ratio cells, but this is simply due to the finer mesh spacing near the source.

Because PWL produces an SPD matrix, we can use the Conjugate Gradient (CG) method to solve the matrix system. Palmer's method must be solved using an asymmetric solver such as GMRES. We expect that CG will be more efficient, and we test this conjecture on a timedependent "tophat" problem, with the radiation diffusion equation coupled to an energy-balance equation for the material (which the material temperature must satisfy). The tophat problem is a two-material problem, with a high-density region and a low-density tophat region. In figure 9, the red region is the high-density, thick region, and the blue region is the low-density, thin region. While the details are not important for our purposes here, we note that the material opacities are temperature-dependent and the radiation source is a Planckian at the material temperature.
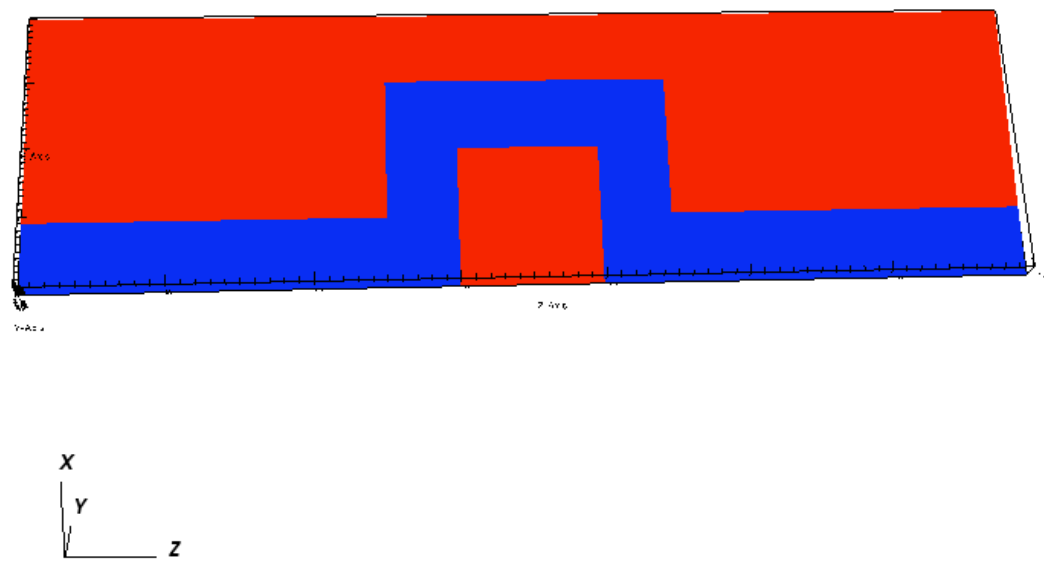

Figure 9: The material densities of the tophat problem

The boundary conditions for this problem are reflecting in the $x$ and $y$ directions and specified intensities in $z$. An incoming intensity is specified on the left boundary in $x$ along the tophat portion (blue material) of the left surface equivalent to a Planckian intensity at $k T=0.3$. Everywhere else on this surface, the value of the boundary condition is vacuum. The initial material temperature is $k T=0.05$ everywhere in the domain, and the simulation is run for 1000 units of time. A few plots of the simulation results are shown in figure 10. These plots were generated using PWL as the diffusion solver. As expected, the radiation flows through the thin region and also eventually "eats" its way into the thick region. This is interesting in itself, but our purpose here is to illustrate the performance of CG and GMRES solvers. 

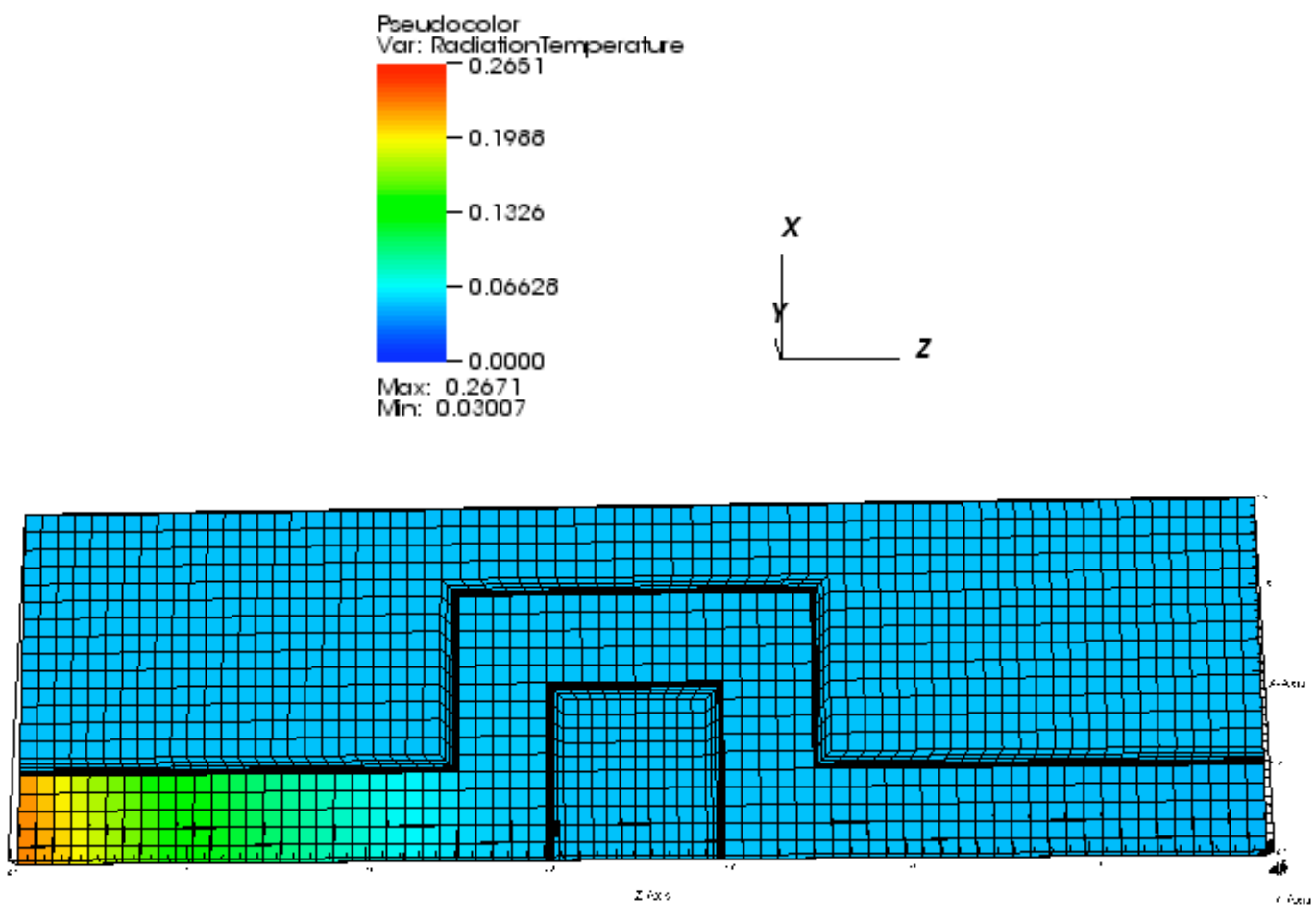

Time $=0.0126066$

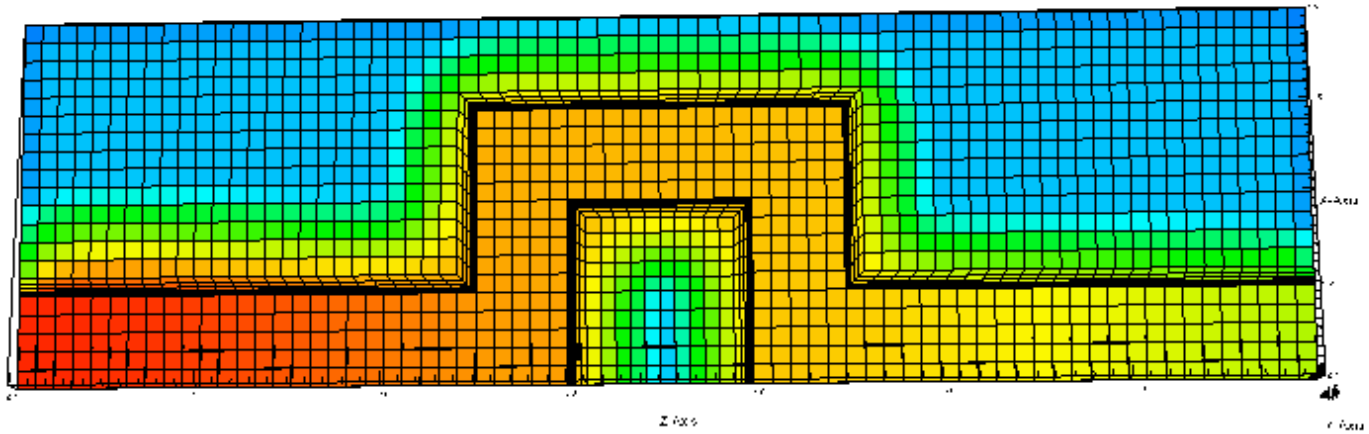

Time $=348.523$

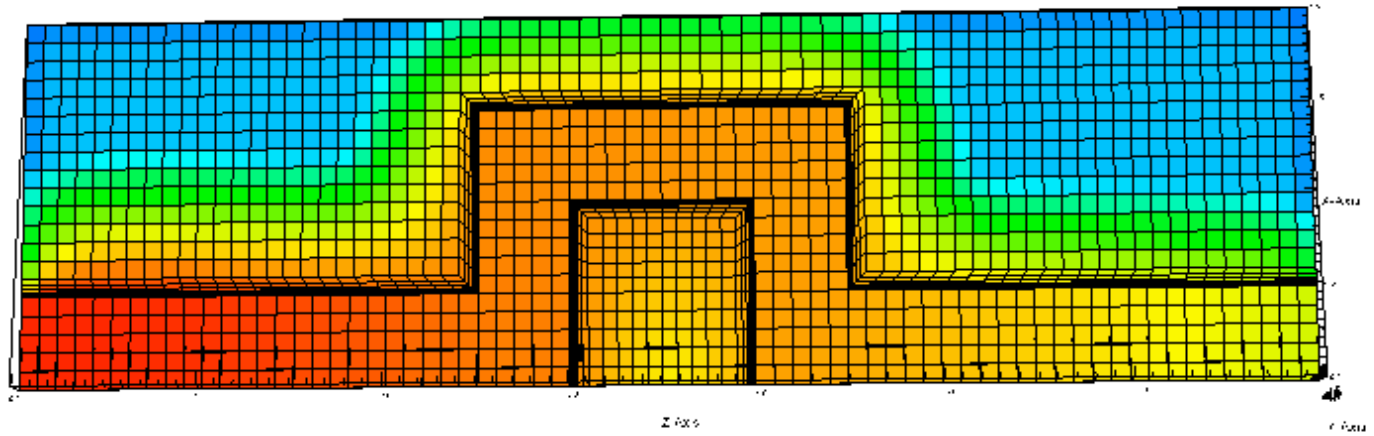

Time $=678.523$ 


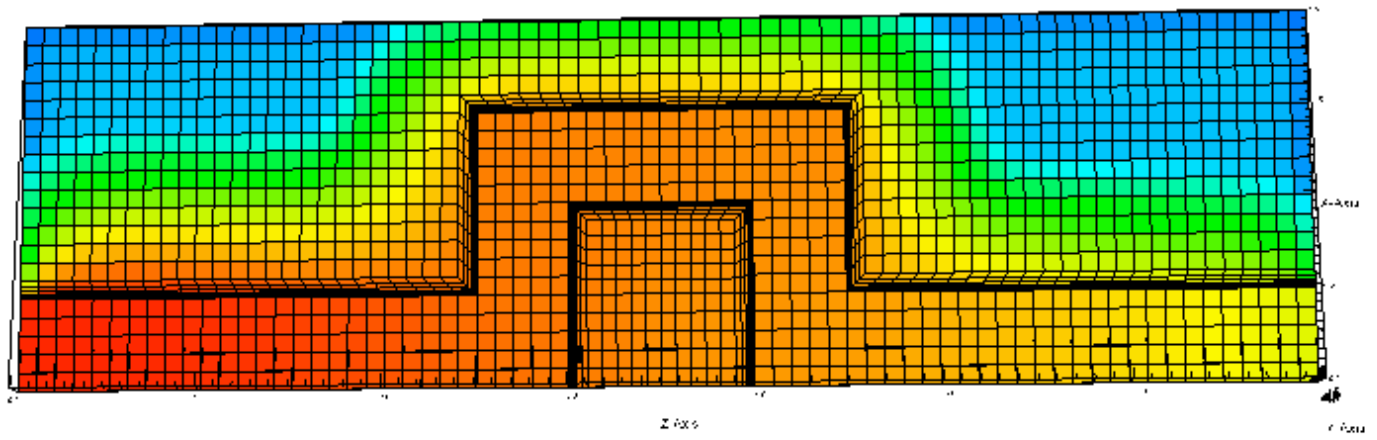

Time $=1000$

Figure 10: The radiation temperature in the tophat problem shown at different time steps.

We compared the effectiveness of both iterative methods (GMRES for Palmer and PWL, and CG for PWL) by comparing the number of iterations required to invert the matrix for each reported time step. The preconditioner used for all linear solvers was Algebraic Multigrid.

The results of these calculations, shown in figure 11, show that CG for PWL requires a factor of three fewer iterations than does GMRES for Palmer's method. (Also, in general GMRES applied to PWL requires slightly fewer iterations than GMRES applied to Palmer's method, which indicates that the PWL matrix is slightly better conditioned than that of Palmer's method.) We further note that in a parallel computing environment, $\mathrm{CG}$ has even more advantages over GMRES, because it requires far fewer inner products per iteration, and each inner product requires global communication. The combination of reduced storage for the matrix, far fewer iterations, reduced storage of solution-length vectors, and fewer inner products per iteration should make the Galerkin PWL method substantially more efficient than Palmer's method.

\section{Iterations counts ( 1 processor, tol=1e-8)}

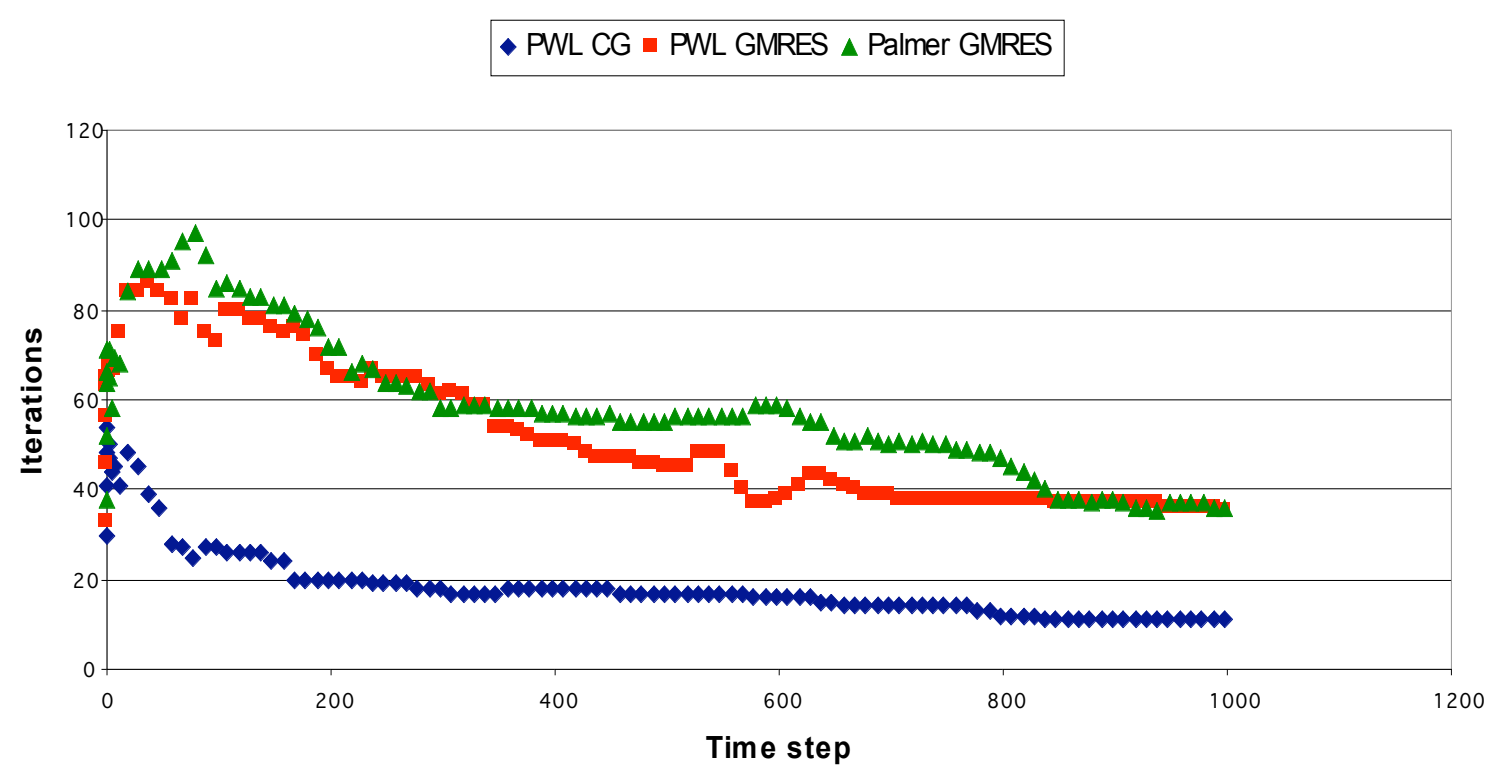

Figure 11: Linear solver comparisons for PWL and Palmer's method for 1 processor. 


\section{CONCLUSIONS}

Our tests show that the Galerkin PWL finite element method applied to the radiation diffusion equation on arbitrary polyhedral meshes has great potential for computational improvements over previous methods. As we had hoped, the PWL method produces results and behaviors almost identical to those of Palmer's method. Palmer's method has a slight theoretical advantage on orthogonal ("brick") grids in that it generates a highly robust 7-point discretization whereas PWL generates full 27-point coupling. However, our tests on problems with very high-aspectratio cells did not uncover any poor PWL behavior. Further, the PWL coefficient matrix is SPD with allows for less storage and much more computationally efficient solutions. We conclude that the Galerkin PWL method is a very attractive option for solving diffusion problems on unstructured grids.

\section{ACKNOWLEDGMENTS}

The research described here was supported in part under the auspices of the U.S. Department of Energy by UC, Lawrence Livermore National Laboratory under Contract No. W-7405-ENG-48.

\section{REFERENCES}

1. T.S. Palmer, "Discretizing the diffusion equation on unstructured polygonal meshes in two dimensions," Annals of Nuclear Energy, 28, pp. 1851-1880, 2000.

2. T.S. Palmer, "A Point-Centered Diffusion Differencing for Unstructured Meshes in 3-D," Proc. Int. Conf. Mathematics and Computations, Reactor Physics and Environmental Analyses, Portland, OR, April 30-May 4, 1995, Vol. 2, pp. 897-905.

3. E.L. Wachspress, A Rational Finite Element Basis, Academic Press, New York (1975).

4. H.G. Stone and M.L. Adams, "A Piecewise Linear Finite Element Basis with Application to Particle Transport," Nuclear Mathematical and Computational Sciences Meeting, Gatlinburg, TN, CD-ROM, April 6-11, 2003

5. J.E. Morel, "A 3-D Cell-Centered Diffusion Discretization for Arbitrary Polyhedral Meshes," Los Alamos National Laboratory Research Note, CCS-4:02-40(U) (2002).

6. Yu. Kuznetsov, K. Lipnikov, M. Shashkov, "Mimetic Finite Difference Method on Polygonal Meshes," Los Alamos National Laboratory Report, LA-UR-03-7608 (2003). 